\title{
ADENOSQUAMOUS CARCINOMA OF THE PANCREAS: A CASE REPORT
}

\author{
Keisha Brooks, \\ $M S, C T(A S C P) M B$ \\ University of Tennessee Health Science Center \\ kbrook13@uthsc.edu \\ David W. Mensi, \\ $B S, S C T(A S C P)$ \\ Trumbull Laboratory \\ dmensi@comcast.com
}

There are no institutional, personal, or financial conflicts of interest associated with this submission.

\begin{abstract}
:
Adenosquamous carcinoma of the pancreas is a rare and aggressive variant of ductal adenocarcinoma which presents both glandular and squamous morphologic features. A cytologic diagnosis of adenosquamous carcinoma on fine needle aspirate preparations is dependent on the identification of both malignant glandular and squamous components. Diagnosis of the malignancy is not particularly difficult if both glandular and squamous components are abundant; however, diagnostic challenges may occur when there is scant cellularity of one of the components, or if one of the components is absent. Because a primary squamous carcinoma of the pancreas is non-existent, a careful search and identification of glandular malignant cells is essential in cases that are squamous dominant.

In this report a case of adenosquamous carcinoma is presented in which a fine needle aspirate was performed. The cytologic and histologic features are described. The cytology findings showed a two cell population of malignant squamous and glandular cells. The histologic findings also showed both glandular and squamous malignant cells, thus confirming the diagnosis of adenosquamous carcinoma.
\end{abstract}

Keywords: Pancreas, Adenosquamous Carcinoma, Mucoepidermoid Carcinoma, Andenoacanthoma 


\section{CASE REPORT}

The patient is a 41 year old African American male with no family history of pancreatic cancer. The patient has a history of hypertension and obesity, but denied any history of smoking or alcohol use. Clinical symptoms include jaundice, nausea, and dark colored urine. A 100 pound weight loss was also reported in the initial diagnosis.

A fine needle aspirate of the pancreas was performed, in which $30 \mathrm{~mL}$ of reddish fluid and multiple string-like tissue fragments were collected and placed in Cytolyte solution. One Thin Prep preparation was prepared along with one cell block preparation. The results of the pre-operative fine needle aspirate were suggestive of adenosquamous carcinoma.

A CT scan showed the location of the tumor in the head of the pancreas. It was identified as large, approximately 7 $\mathrm{cm}$, invading the duodenum and in close proximity to the portal vein. There was extensive perineural invasion. The tumor also directly invaded into the pyloric antrum. A 12-hour pancreaticoduodenectomy was performed. The patient recovered from surgery and started adjuvant chemotherapy, but a repeat CT scan showed diffuse liver metastasis. The patient then began to receive palliate chemotherapy with cisplatin and gemcitabine. The patient was later switched to folfirinox due to the lack of response to the previous chemotherapy treatments.

The pancreaticoduodenectomy produced several specimens for pathologic diagnosis. The following histologic specimens were submitted: 1) Gallbladder, 2) Pancreas, and 3) Duodenum.

\section{CYTOLOGIC FINDINGS}

The FNA produced a cellular specimen consisting of a two cell population of both glandular squamous malignant cells. The malignant glandular cells are composed of crowded and disorganized nuclei in which prominent anisonucleosis is present (Figure 1). The nuclei have irregular nuclear membranes and bland evenly distributed chromatin. Prominent nucleoli were also observed, and hyperchromasia was minimal. The cytoplasm had a foamy and vacuolated consistency, which is characteristic of glandular cells. Figures 2 and 3 show malignant squamous cells, with hyperchromatic nuclei and coarse chromatin. In comparison to the cytoplasm of glandular cells, the malignant squamous cells have cytoplasm that is absent of vacuolization, and is denser in appearance.

\section{HISTOLOGIC FINDINGS}

The gallbladder specimen showed moderately severe chronic cholecystitis and patchy moderate cholesterolosis, with no evidence of malignancy identified. Histologic findings from the pancreas revealed moderately differentiated adenosuqmous carcinoma of the pancreas $7 \mathrm{~cm}$ in size, involving most of the pancreatic head and uncinate process. The tumor produced a large elongated 10x4 cm duodenal ulcer that extended into the pyloric antrum, just above the ampulla of Vater, which represented extrapancreatic direct invasion. The ampulla of Vater was intruded by a pushing margin of the tumor, but did not show invasion into the mucosa. Four of twenty-six peripancreatic lymph nodes were positive for malignancy. Two perigastric lymphnodes were negative for 
metastasis. The gastric antrum showed moderately severe active chronic atrophic gastritis, but no evidence of metastasis.

The histology figures presented in this case report are of the pancreas tissue specimen. Figure 4 shows the presence of malignant glands that are in close proximity of one another. The cells in the glands are columnar in shape with luminous spaces. Figure 5 is a close-up view of one of the glands, which illustrates the presence of glandular cells with eccentrically placed nuclei and foamy vacuolated cytoplasm. The nuclei show irregular nuclear membranes, moderate hyperchromasia, coarse chromatin, and prominent nucleoli. Another area of the pancreas revealed malignant squamous cells, as shown in Figures 6 and 7. Figure 6 shows a flat sheet of malignant cells, with squamous pearl formation. Figure 7 shows malignant squamous cells with multinucleation, coarse chromatin, and prominent nucleoli. The cytoplasm also has a thick consistency, when compared to the cytoplasm of malignant glandular cells.

\section{CONCLUSION}

In conclusion, adenosquamous carcinoma of the pancreas is a rare and aggressive tumor with a poor prognosis. The diagnosis of the tumor depends on the identification of both malignant squamous and glandular cells. The reported case had a sufficient amount of both malignant components for an accurate diagnosis, and the histologic findings further substantiated the diagnosis. No patient follow-up or life expectancy outcomes were available for the patient. It is known that the patient was given palliative chemotherapy treatment after the discovery of distant metastasis to the liver.

\section{DISCUSSION}

Adenosquamous carcinoma, also known as mucoepidermoid carcinoma and adenoacanthoma is a rare variant of ductal adenocarcinoma of the pancreas ${ }^{1,2}$. It accounts for $1-4 \%$ of all exocrine pancreatic malignancies ${ }^{3,4,5}$. Due to its rare incidence, there is a scarcity of literature in regards to the correlation of clinical features and patient outcomes. Common symptoms of adenosquamous carcinoma include abdominal pain, jaundice, weight loss, and anorexia ${ }^{6,7}$. The prognosis for individuals with the malignancy is less promising than the conventional pancreatic ductal adenocarcinoma, with a survival rate of less than one year after surgery ${ }^{8}$.

Reported cases of pancreatic adenosquamous carcinoma show a higher incidence in males over the age of sixty ${ }^{3}$. However, a study conducted by Hsu et al. of twelve cases of the disease found more women affected by the malignancy with a mean age of 71 years ${ }^{6}$. Unlike other findings in the literature that cite the head of the pancreas as the common location of the tumor ${ }^{3}$, Hsu et al also reported an even dissemination of the tumor in the head, body and tail of the organ ${ }^{6}$.

Cytologically, the squamous component of the tumor consists of cells with mostly eosinophilic cytoplasm and occasional squamous pearl formations ${ }^{9}$. Malignant squamous tumor cells may also show varying degrees of cellular pleomorphism with an increased grade of the tumor. Malignant squamous cells can appear as single lying cells, or in abnormal syncytial groups with orangeophilic or basophilic cytoplasm ${ }^{10}$. The glandular component shows variability of nuclear size and shape, with a higher degree of nuclear hyperchromasia, nuclear 
enlargement, coarse chromatin, and prominent nucleoli with an increase in tumor the stage of the tumor. The cells have foamy vacuolated cytoplasm with eccentrically placed nuclei.

The abundance of squamous component varies with this malignancy, and in some cases may present itself as a pure squamous carcinoma ${ }^{11}$. Because a primary squamous carcinoma of the pancreas is non-existent, is it important to identify the glandular component of the tumor to accurately diagnose adenosquamous carcinoma. Likewise, it is also necessary to rule out a metastatic pulmonary squamous carcinoma of the pancreas in cases of a scant glandular component $^{12,13}$. It is imperative to correlate cytologic and histologic findings along with clinical history for proper patient management and treatment.

Surgical resection is the customary treatment for adenosquamous carcinoma of the pancreas ${ }^{14,15}$. In a study of 415 patients with adenosquamous carcinoma, Boyd et al. ${ }^{15}$ found patients with the malignancy were more likely to undergo surgical resection than those with pancreatic ductal adenocarcinoma. In spite of the less than favorable prognosis of adenosquamous carcinoma, surgical resection offers the patient a slightly higher chance of long term survival.

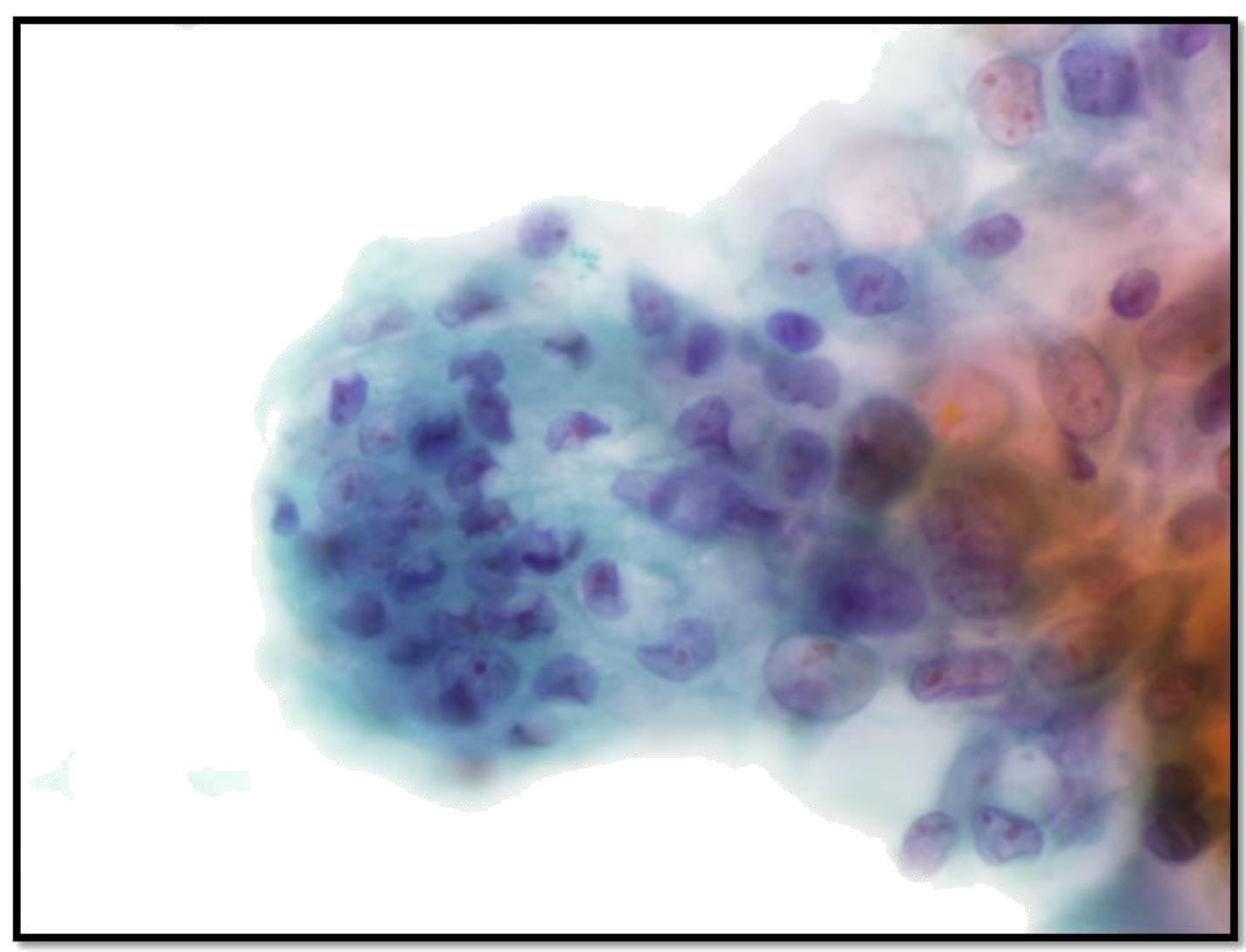

Figure 1: 60x magnification. Malignant glandular cells containing crowded nuclei exhibiting anisonucleosis, bland chromatin, and prominent nucleoli. Cytoplasm is foamy and vacuolated. 


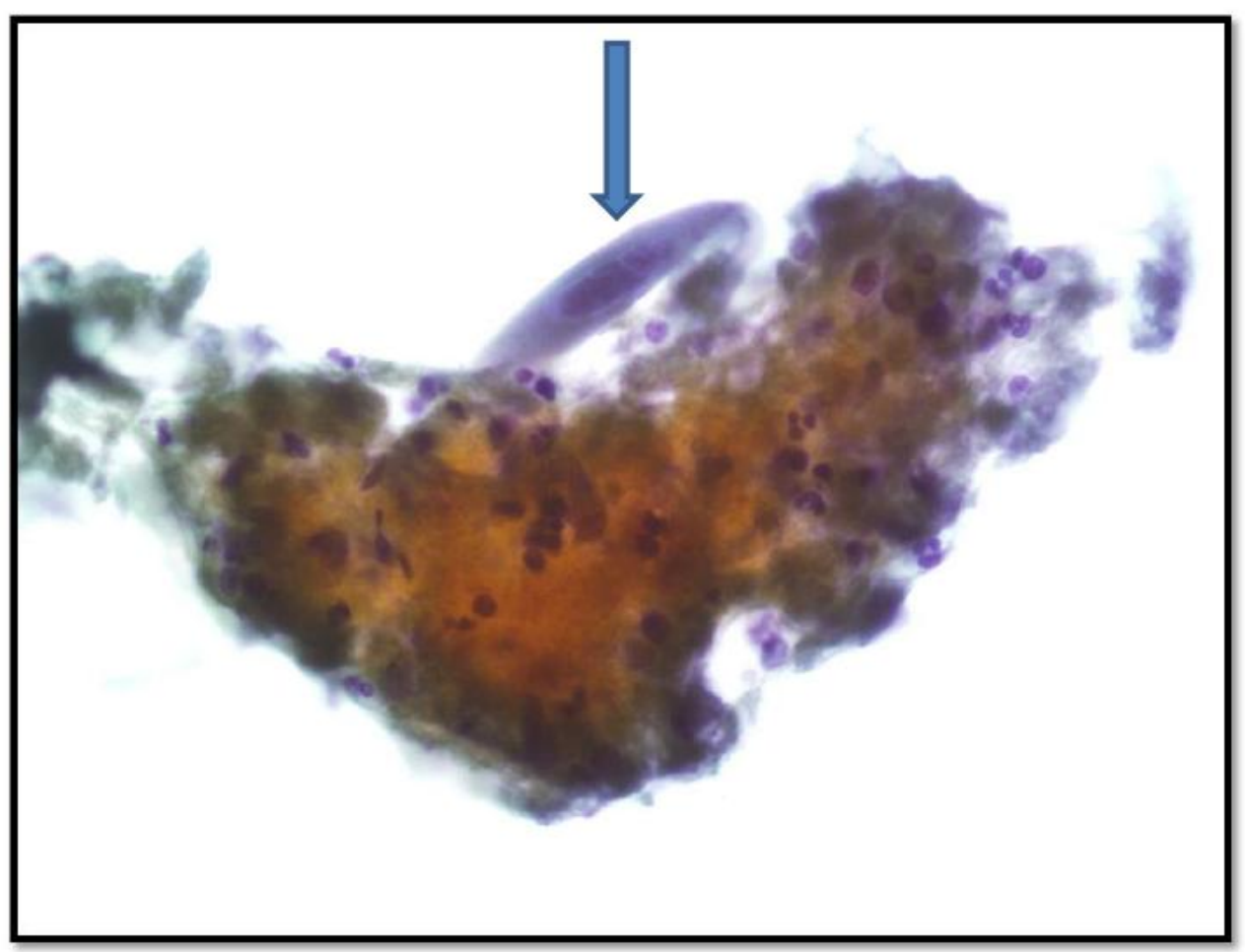

Figure 2: 40x magnification. Malignant squamous cell with dense cytoplasm (arrow). Nucleus is hyperchromatic with coarse chromatin.

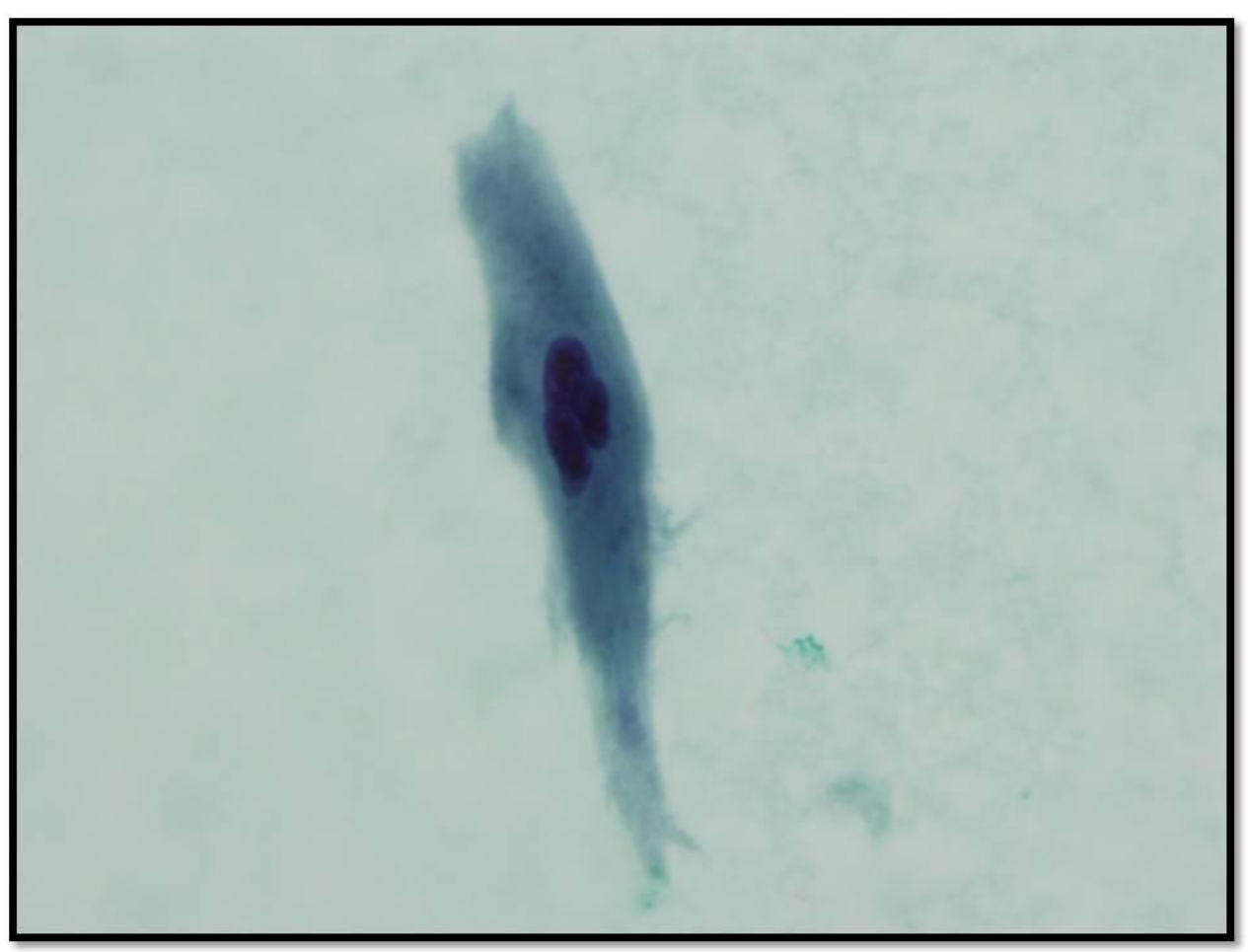

Figure 3: Malignant squamous cell with multinucleation. Nuclei are hyperchromatic with coarse chromatin. Cytoplasm has thick consistency and pleomorphic shaped. 


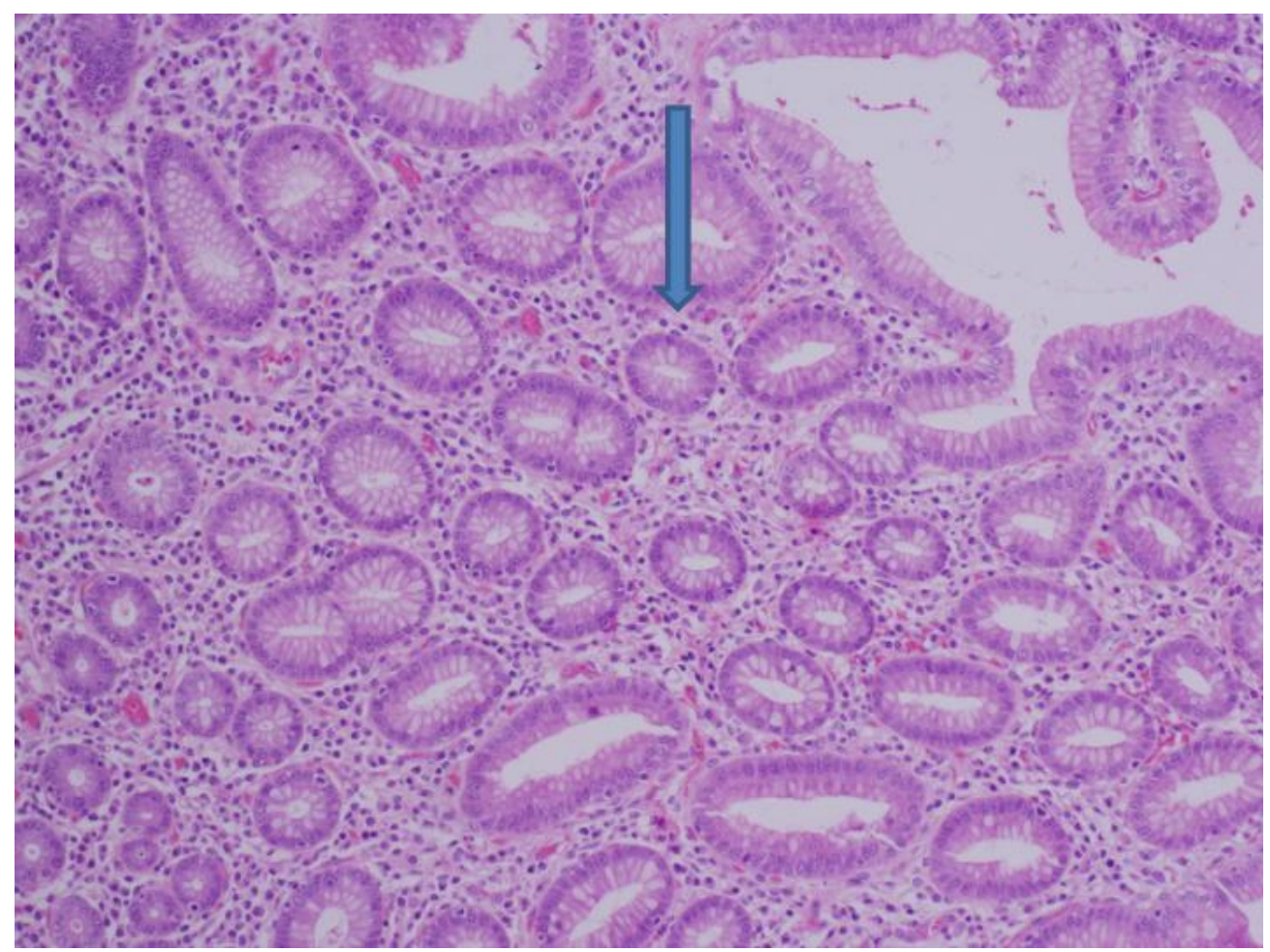

Figure 4: Histology section, pancreas (20x magnification). Glandular cells with columnar formations, luminous spaces, and eccentrically placed nuclei.

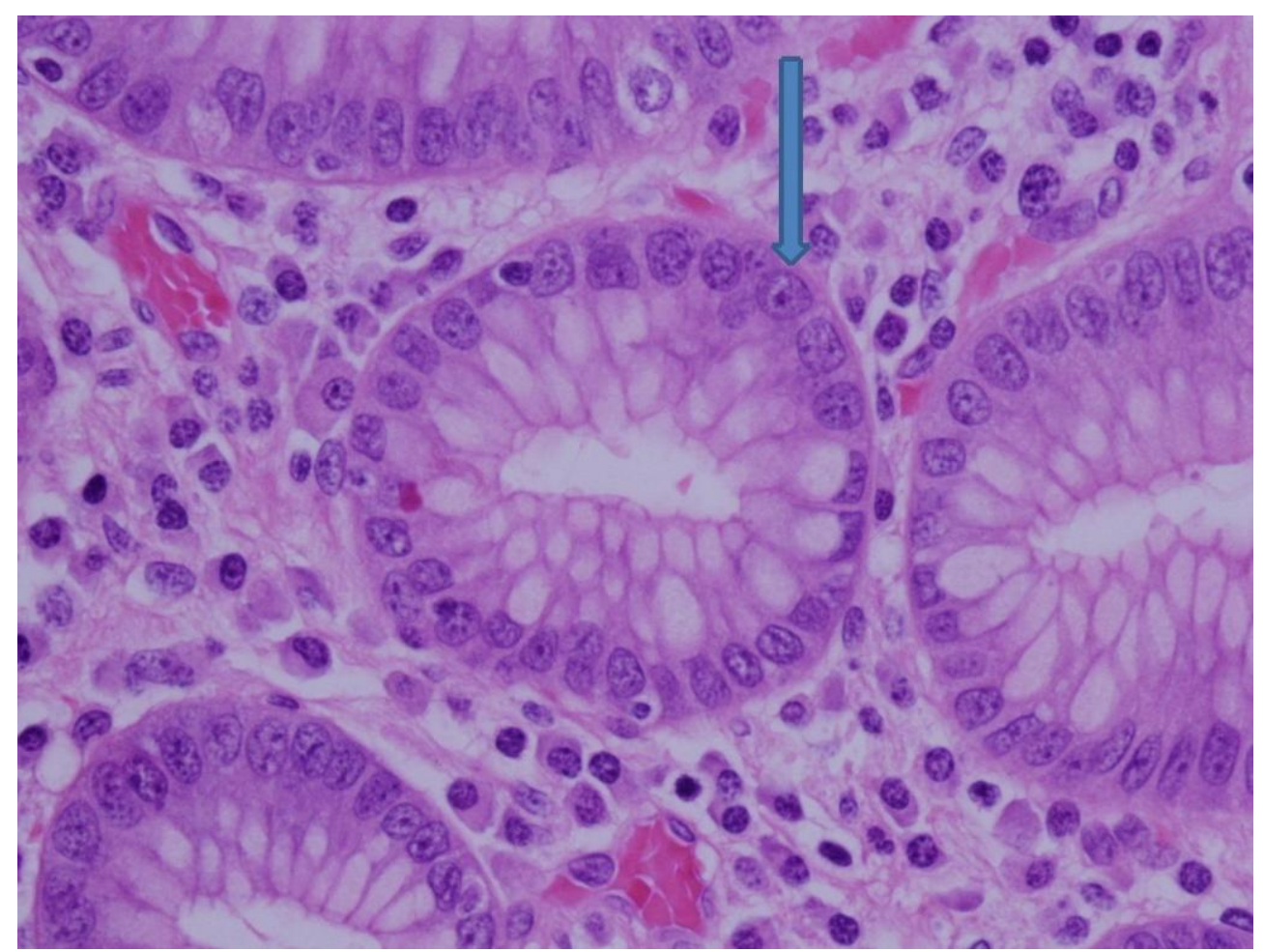

Figure 5: 60x magnification, pancreatic gland. Malignant cells with eccentrically placed nuclei, coarse chromatin, and prominent nucleoli (arrow). Cytoplasm is vacuolated and columnar in shape. 


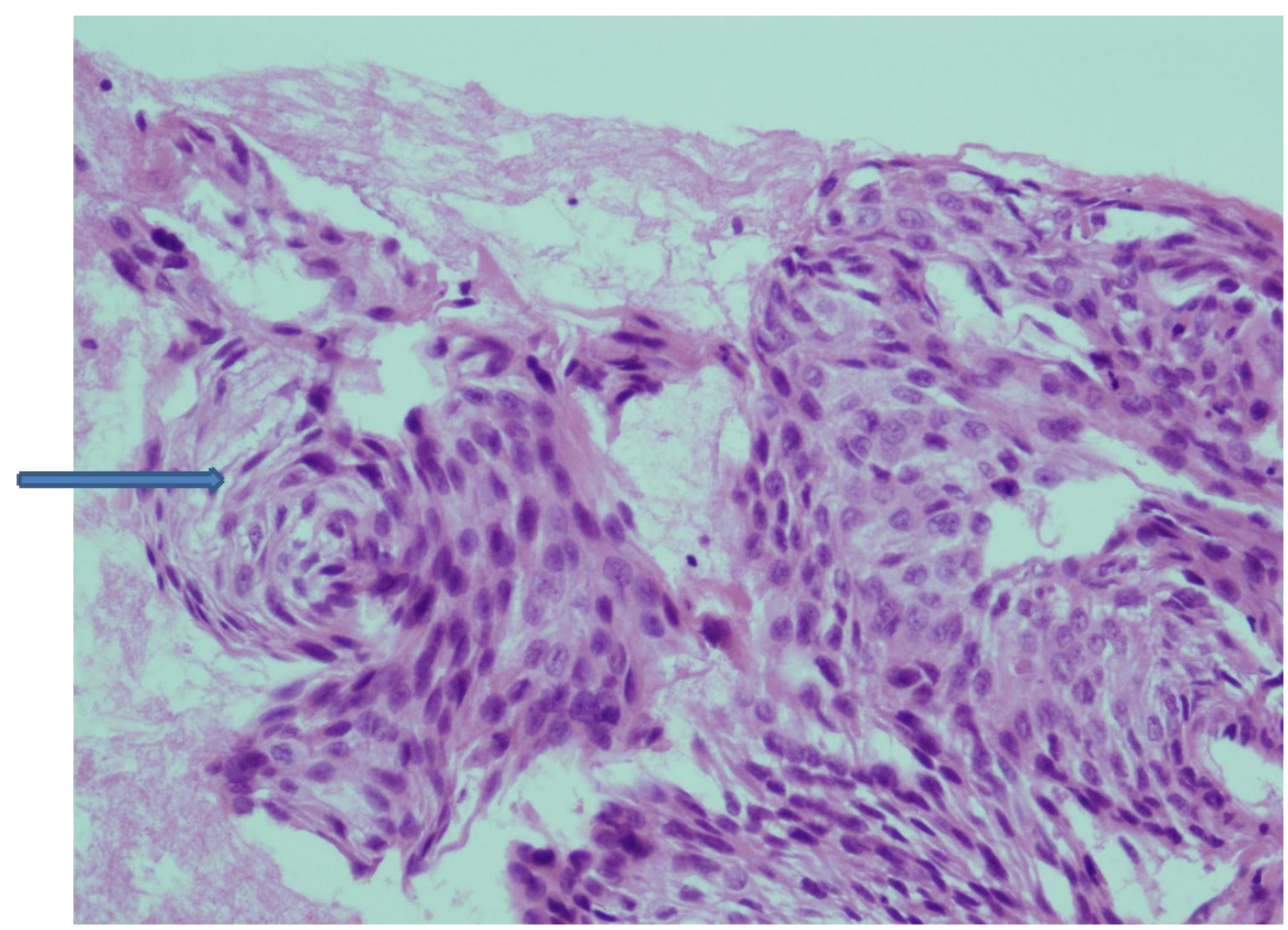

Figure 6: 20x magnification. Malignant sheet of squamous cells. Pearl formation, a swirling of squamous cells, is observed at the far left (arrow). Nuclei have coarse chromatin and prominent nucleoli.

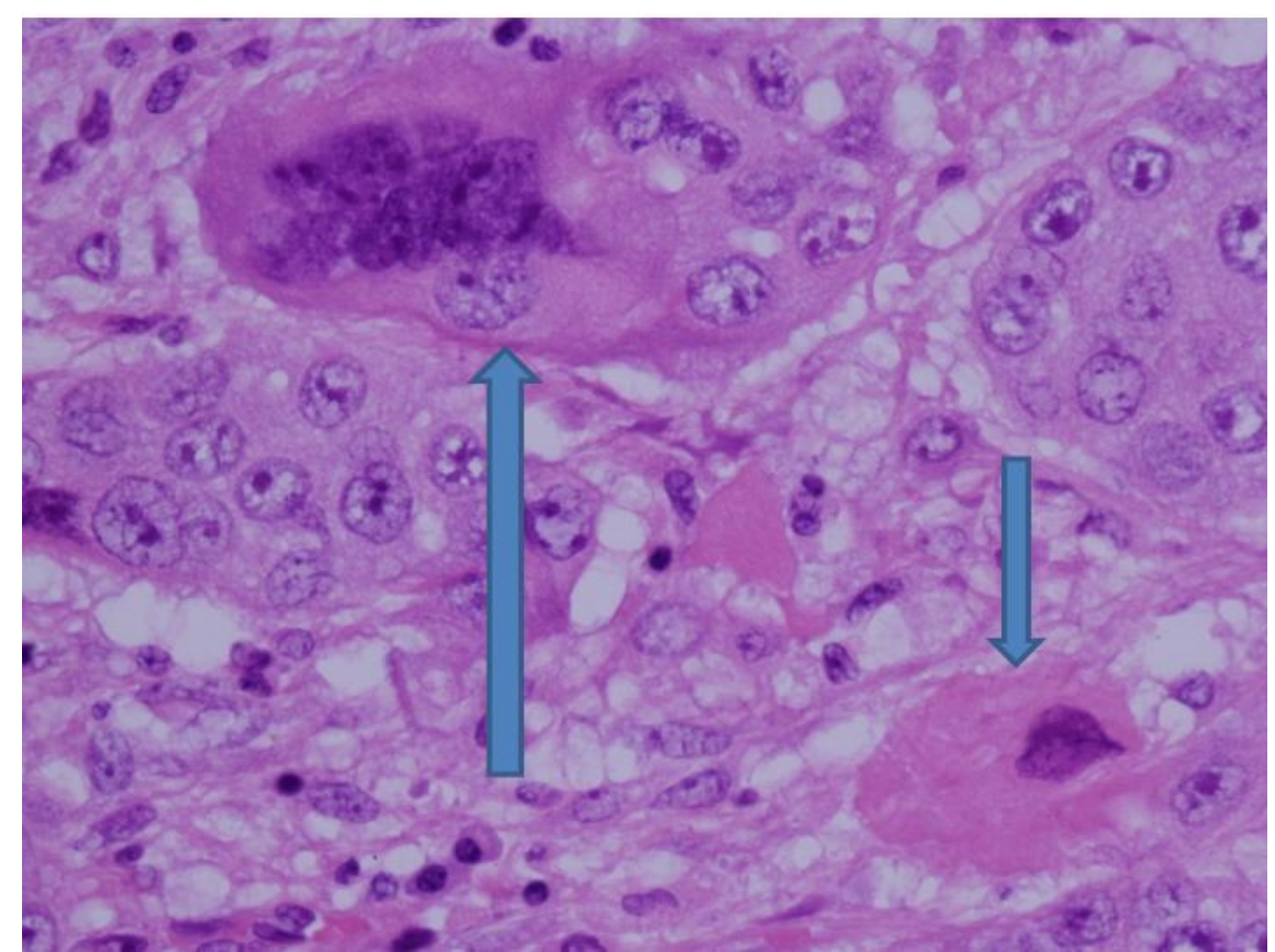

Figure 7: 40x magnification. Malignant squamous cells with dense cytoplasm, multinucleation, and pleomorphic nuclei. Nuclei contains prominent nucleoli. 


\section{REFERENCES}

1. Cihak, Robert W., Taketsugu Kawashima, and Arthur Steer. "Adenoacanthoma (adenosquamous carcinoma) of the pancreas." Cancer 29, no. 5 (1972): 1133-1140.

2. Sommers, Sh C., and W. A. Meissner. "Unusual carcinomas of the pancreas." AMA archives of pathology 58, no. 2 (1954): 101-111..

3. Madura, James A., Benjamin $T$. Jarman, Michael G. Doherty, Moo-Nahm Yum, and Thomas J. Howard. "Adenosquamous carcinoma of the pancreas." Archives of Surgery 134, no. 6 (1999): 599-603.

4. Cubilla, Antonio L., and Patrick J. Fitzgerald. "Morphological patterns of primary nonendocrine human pancreas carcinoma." Cancer research 35 , no. 8 (1975): 2234-2246.

5. Fitzgerald, Timothy L., Zach J. Hickner, Matthew Schmitz, and Eric J. Kort. "Changing incidence of pancreatic neoplasms: a 16-year review of statewide tumor registry." Pancreas 37, no. 2 (2008): 134-138.

6. Hsu, Jun-Te, Han-Ming Chen, RenChin Wu, Chun-Nan Yeh, Ta-Sen Yeh, Tsann-Long Hwang, Yi-Yin Jan, and Miin-Fu Chen. "Clinicopathologic features and outcomes following surgery for pancreatic adenosquamous carcinoma." World J Surg Oncol 6, no. 95 (2008): 531535 .

7. Okabayashi, Takehiro, and Kazuhiro Hanazaki. "Surgical outcome of adenosquamous carcinoma of the pancreas." World journal of gastroenterology: WJG 14, no. 44 (2008): 6765.

8. Hsu, J. T., C. N. Yeh, Y. R. Chen, H. M. Chen, T. L. Hwang, Y. Y. Jan, and M.
F. Chen. "Adenosquamous carcinoma of the pancreas." Digestion 72, no. 2-3 (2004): 104-108.

9. Ishikawa, Osamu, Yukio Matsui, Ikutoshi Aoki, Takeshi Iwanaga, Toshio Terasawa, and Akira Wada. "Adenosquamous carcinoma of the pancreas: a clinicopathologic study and report of three cases." Cancer 46, no. 5 (1980): 1192-1196.

10. Renshaw, Andrew. "Silverberg's Principles and Practice of Surgical Pathology and Cytopathology." (2006): 203.

11. Itani, Kamal MF, Amir Karni, and Linda Green. "Squamous cell carcinoma of the pancreas." Journal of Gastrointestinal Surgery 3, no. 5 (1999): 512-515.

12. Mockli, Gary C., and Mark -Silversmith. "Squamous cell carcinoma of the lung metastatic to the pancreas: Diagnosis by fine needle aspiration biopsy." Diagnostic cytopathology 16, no. 3 (1997): 287-288.

13. Sidawy, Mary K., and Syed Z. Ali, eds. Fine needle aspiration cytology. Elsevier Health Sciences, 2007.

14. Kardon, David E., Lester DR Thompson, Ronald M. Przygodzki, and Clara S. Heffess. "Adenosquamous carcinoma of the pancreas: a clinicopathologic series of 25 cases." Modern Pathology 14, no. 5 (2001): 443451.

15. Boyd, Casey A., Jaime BenarrochGampel, Kristin M. Sheffield, Catherine D. Cooksley, and Taylor S. Riall. "415 patients with adenosquamous carcinoma of the pancreas: a population-based analysis of prognosis and survival." Journal of Surgical Research 174, no. 1 (2012): 1219. 